\title{
Distinct clinicopathologic factors and prognosis based on the presence of ground-glass opacity components in patients with resected stage I non-small cell lung cancer
}

\author{
Chengdi Wang', Yuxuan Wu ${ }^{1}$, Jingwei $\mathrm{Li}^{1}$, Pengwei Ren ${ }^{1}$, Ya Gou ${ }^{1}$, Jun Shao ${ }^{1}$, Yaojie Zhou ${ }^{1}$, Xue Xiao ${ }^{1}$, \\ Paierhati Tuersun ${ }^{1}$, Dan Liu ${ }^{1}$, Li Zhang ${ }^{2}$, Weimin Li $^{1}$ \\ ${ }^{1}$ Department of Respiratory and Critical Care Medicine, ${ }^{2}$ Precision Medicine Center, West China Hospital, West China Medical School, Sichuan \\ University, Chengdu, China \\ Contributions: (I) Conception and design: W Li, L Zhang; (II) Administrative support: W Li, D Liu; (III) Provision of study materials or patients: \\ All authors; (IV) Collection and assembly of data: C Wang, Y Wu, J Li, P Ren, Y Gou, J Shao, Y Zhou, X Xiao, P Tuersun; (V) Data analysis and \\ interpretation: C Wang, Y Wu; (VI) Manuscript writing: All authors; (VII) Final approval of manuscript: All authors. \\ Correspondence to: Li Zhang, PhD. Precision Medicine Center, West China Hospital, West China Medical School, Sichuan University, No. 37 Guoxue \\ Xiang, Chengdu, China. Email: zhangli7375@scu.edu.cn; Weimin Li, PhD. Department of Respiratory and Critical Care Medicine, West China \\ Hospital, West China Medical School, Sichuan University, 37 Guoxue Xiang, Chengdu, China. Email: weimi003@scu.edu.cn.
}

Background: This study was to investigate the prognostic value of ground-glass opacity(GGO) components and to evaluate distinct the clinicopathological variables of survival outcomes for the pureGGO, part-solid and solid groups of patients with resected stage I non-small cell lung cancer (NSCLC).

Methods: We retrospectively reviewed the structured data for stage I NSCLC patients who had undergone the curative-intent surgical resection in the Lung Cancer Database of West China Hospital from 2009 to 2016. The eligible patients were divided into the pure-GGO, part-solid and solid groups according to the radiological manifestation. Univariate and multivariate Cox regression analyses were performed between the 3 groups. And we further evaluated the clinicopathological variables in each group separately.

Results: Among a total of 2,775 eligible patients enrolled into the cohort were 1,587 (57.19\%) in the solid group, $508(18.31 \%)$ in the part-solid group, and $680(24.50 \%)$ in the pure-GGO group. The 5-year overall survival (OS) and recurrence-free survival (RFS) rates were $98.8 \%$ and $98.0 \%$ in the pure-GGO group, $96.0 \%$ and $86.5 \%$ in the part-solid group, and $88.0 \%$ and $75.5 \%$ in the solid group, respectively $(\mathrm{P}<0.001)$. Presence of GGO components was a significantly favorable prognosticator $(\mathrm{HR}=0.415,95 \% \mathrm{CI}$ : 0.286-0.601). Different groups had distinct prognostic factors. LVI was the shared risk factor for groups with presence of GGO components in both part-solid and pure-GGO groups. Pathological stage (IA or IB) was influential exclusively for the pure-GGO group. In the solid group, females, younger patients, and patients without VPI had better survival. But such independent significance did not exist in the other two groups.

Conclusions: GGO component was a strong prognosticator of better prognosis in resected patients with stage I NSCLC. Prognostic factors and survival outcomes were disparate among the pure-GGO, partsolid, and solid group. Our results support the proposal that the next edition tumor-node-metastasis (TNM) classification should consider the importance of GGO components as a new T descriptor.

Keywords: Non-small cell lung cancer (NSCLC); ground-glass opacity (GGO); part-solid nodule; solid nodule; survival analysis

Submitted May 20, 2020. Accepted for publication Aug 20, 2020.

doi: 10.21037/atm-20-4971

View this article at: http://dx.doi.org/10.21037/atm-20-4971 


\section{Introduction}

Lung cancer has the highest incidence of all cancers (11.6\% of all new cases) and is the leading cause of cancer-related mortality (18.4\% of all cancer deaths) worldwide. In 2018, an estimated 2.09 million new cases of lung cancer and 1.76 million lung cancer related deaths are projected to occur throughout the world, while $37.0 \%$ new cases and $39.2 \%$ deaths were estimated to occur in China (1). Despite the rapid development of diagnosis and treatment modalities, the 5-year overall survival (OS) rate of lung cancer remains poor with in China at $19.8 \%$ and in the USA at $21.2 \%$. This disappointing OS rate can be explained by the fact that approximately $70 \%$ of patients are diagnosed at the advanced stage $(2,3)$. If lung cancer can be detected and diagnosed at an earlier stage, when there have not been lymphatic and distant metastases, the prognosis of lung cancer patients will be notably favorable. Compared with chest radiography (CR), computed tomography (CT) scans were more effective in the detection of lung cancer and could reduce the mortality rate by $20.0 \%$ or more if adopted annually, which was validated by the U.S.-based National Lung Screening Trial (NLST) and the DutchBelgian-based Nederlands-Leuvens Longkanker Screenings On-derzoek (NELSON) (4-6).

Tumor-node-metastasis (TNM) staging is seem crucial for lung cancer patients. But the current staging criteria need to be updated. According to the eighth of TNM classification, tumor diameter is determined based on pathological or radiological findings (7-9). But aside from diameter, more characteristics should be taken into account. According to the radiomic features shown in chest CT scans, lung nodules can be classified into a pure ground-glass (pure-GGO) group with only GGO components, a solid group, and a part-solid group with both GGO and solid components (10-12). Recent studies have found that the presence of GGO components in lung nodules was significantly associated with the encouragingly prolonged survival outcomes (13-15). Lung cancer patients with pure-GGOs had better 5-year recurrencefree survival (RFS) and overall survival (OS) compared with those with part-solid nodules, and patients with pure solid tumors had even worse outcomes (16). More emphases should be placed on the prognostic importance of these radiomic features, which is neglected by current classification criteria.

To further investigate the effects of the presence and the proportion of GGO components, this research identified a large cohort of patients with resected stage I non-small cell lung cancer (NSCLC) and divided patients into 3 groups labeled as pure-GGO, part-solid, and pure solid respectively. Differences in clinicopathologic variables and survival were evaluated between the 3 groups.

We present the following article in accordance with the STROBE reporting checklist (available at http://dx.doi. org/10.21037/atm-20-4971).

\section{Methods}

\section{Patient selection}

We retrospectively searched lung cancer database of West China Hospital of Sichuan University for resected stage I patients between 2009 and 2016 according to the 8 th edition of the American Joint Committee (AJCC) on Cancer Staging Manual. Patients who had undergone surgical resection with preoperative thoracic CT scans and who had been pathologically diagnosed with lung cancer were identified as eligible and included in our study. Patients who harbored multi-focal tumors such as synchronous/ metachronous lung cancers, or those who had no available preoperative CT scans were excluded.

This study was approved by the Institutional Review Board of West China Hospital of Sichuan University. The study was conducted in accordance with the Declaration of Helsinki (as revised in 2013). Requirements of informed consent were waived due this study's retrospective nature.

\section{Patient characteristics}

The retrieved medical record data included the following characteristics: sex, age, tumor family history, smoking history, tumor subtype, pathologic TNM stage, visceral pleural invasion (VPI), lymphovascular invasion (LVI). Radiological images were obtained from the picture archiving and communication system (PACS) and the grouping procedure was completed by two experienced staffs independently. Any disagreements should be carefully reconsidered and resolved by a consensus.

Tumor subtypes were determined according to the International Association for the Study of Lung Cancer (IASLC)/the American Thoracic Society (ATS)/the European Respiratory Society (ERS) and the 2015 World Health Organization (WHO) classifications. NSCLC was classified into lung adenocarcinoma (LUAD), squamous carcinoma (LUSC), adeno-squamous carcinoma (LASC) and others. 


\section{Follow-up protocol}

Postoperative follow-ups were arranged every 3 months in first 2 years after resection, every 6 months from the third to fifth year, and annually thereafter. Follow-up procedures included regular chest/upper-abdominal CT scans and tumor marker examination. Brain magnetic resonance imaging (MRI), bone scintigraphy, or supraclavicular/ abdominal/cervical ultrasonography were optional and performed when aberrant symptoms of corresponding regions occurred. OS was calculated from initial surgery to the day of last follow-up or death due to any cause. Recurrence-free survival (RFS) was calculated from the initial operation to the time of first recurrence or the last clinical visit.

\section{Statistical analysis}

OS and RFS were analyzed using the Kaplan-Meier method, and the log-rank test was performed to compare the variables between groups. Univariate and multivariate analyses were conducted to identify significant and independent prognostic factors by using SPSS software (version 21.0, IBM Inc., NY, USA) and R software (version 3.6.0). Two-sided $P$ values lower than 0.05 were considered statistically significant.

\section{Results}

\section{Patient characteristics}

A total of 2,775 eligible lung cancer patients met the inclusion criteria and were enrolled into the cohort. Of these patients, $1,587(57.19 \%)$ were placed in the solid group, $508(18.31 \%)$ placed in the part-solid group, and 680 (24.50\%) placed in the pure-GGO group on the basis of radiological manifestation. The patients' clinicopathological characteristics were summarized in Table 1. Statistically significant differences were observed across the three groups regarding the sex $(\mathrm{P}<0.001)$, age $(\mathrm{P}<0.001)$, smoking history $(\mathrm{P}<0.001)$, family history of lung cancer $(\mathrm{P}<0.001)$, pathologic subtype $(\mathrm{P}<0.001)$, p-stage $(\mathrm{P}<0.001)$, LVI $(\mathrm{P}<0.001)$, and VPI $(\mathrm{P}<0.001)$.

The results of clinical variables indicated that, compared with the solid group, females $(68.38 \%$ for the pure-GGO group, $57.68 \%$ for the part-solid group, $43.54 \%$ for the solid group), never-smokers (81.76\% for the pure-GGO group, $70.67 \%$ for the part-solid group, $52.43 \%$ for the solid group), younger age ( $\leq 65$ years) $(61.91 \%$ for the pure-
GGO group, $52.17 \%$ for the part-solid group, $47.45 \%$ for the solid group), LUAD subtype ( $98.97 \%$ for the pureGGO group, $92.72 \%$ for the part-solid group, $70.70 \%$ for the solid group) accounted for a greater percentage in groups with the presence of GGO components (the pureGGO group and part-solid group). Among the 352 patients with LUSC, the second largest subtype of lung cancer, $91.48 \%(\mathrm{n}=322)$ were in the solid group.

\section{Survival outcomes}

The median follow-up period was 58.9 months (IQR: 43.983.2 months). The 5-year OS rate and 5-year RFS rate were $91.7 \%$ and $82.4 \%$ for all the resected stage I NSCLC cases, respectively (Figure 1). The 5-year OS rates were $98.8 \%$ for the pure-GGO group, $96.0 \%$ for the part-solid group, and $88.0 \%$ for the solid group $(\mathrm{P}<0.001)$ (Figure 2$)$. The 5 -year RFS rates were $98.0 \%$ for the pure-GGO group, $86.5 \%$ the part-solid group, and $75.5 \%$ for the solid group $(\mathrm{P}<0.001)$ (Figure 2). These data indicated that the pure-GGO group had a more favorable clinical outcome. Earlier stage and smaller tumor also indicated better survival. According to the p-stage classification, stage I patients included in our analyses were subdivided into 4 subgroups, namely IA1 (T1aN0MO), IA2 (T1bN0MO), IA3 (T1cN0MO), and IB (T2aN0MO). The 5 -year OS rates were $99.2 \%$ for IA1, $95.1 \%$ for IA2, $92.0 \%$ for IA3, and $88.9 \%$ for IB $(\mathrm{P}<0.001)$ (Figure 3). And the 5 -year RFS rates were $96.9 \%$ for IA1, $89.6 \%$ for IA2, $85.5 \%$ for IA3, and $73.6 \%$ for IB $(\mathrm{P}<0.001)$ (Figure 3). With p values both less than 0.001, significant differences were observed regarding 5-year OS and RFS rates between different subgroups.

The univariate and multivariate Cox proportional hazards regression analyses indicated that older age (HR $=1.836$, 95\% CI: 1.424-2.368), VPI (visceral pleural invasion) $(\mathrm{HR}=1.438,95 \% \mathrm{CI}: 1.060-1.951)$, solid components (HR $=4.292,95 \%$ CI: 2.214-8.320) were independently significant prognostic factors for survival on the whole (Table 2).

\section{Distinct prognostic factors in different groups}

The presence of GGO components was a significant prognosticator (HR $=0.415,95 \%$ CI: 0.286-0.601). Furthermore, the univariate and multivariate Cox proportional hazards regression analyses of clinicopathological factors were performed separately in each of the 3 groups (pure-GGO group, part-solid group 
Table 1 Clinicopathologic characteristics of enrolled lung cancer patients with resected stage I NSCLC $(\mathrm{n}=2,775)$

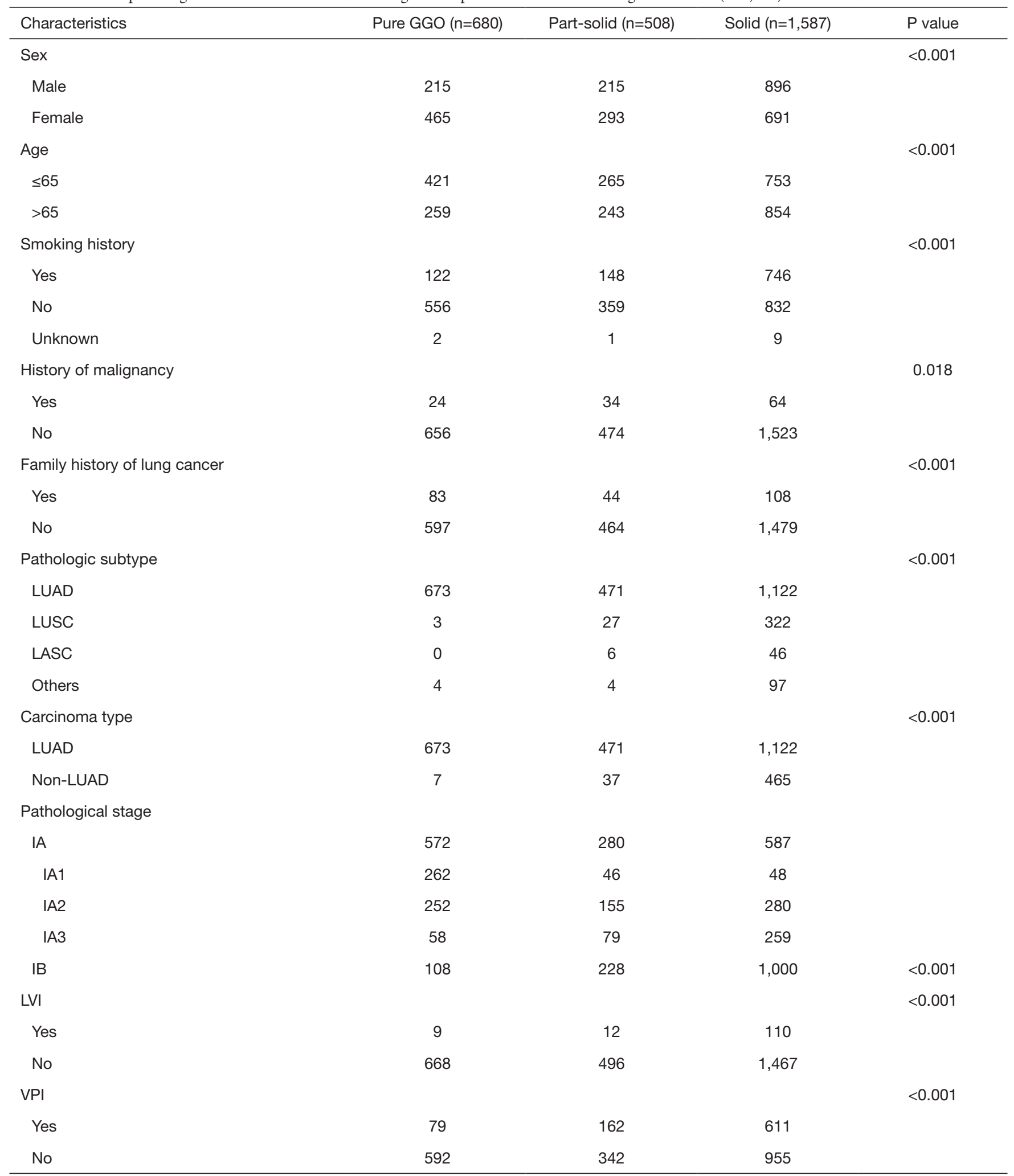

GGO, ground-glass opacity; LASC, lung adenosquamous carcinoma; LVI, lymphovascular invasion; LUAD, lung adenocarcinoma; LUSC, lung squamous carcinoma; VPI, visceral pleural invasion. 

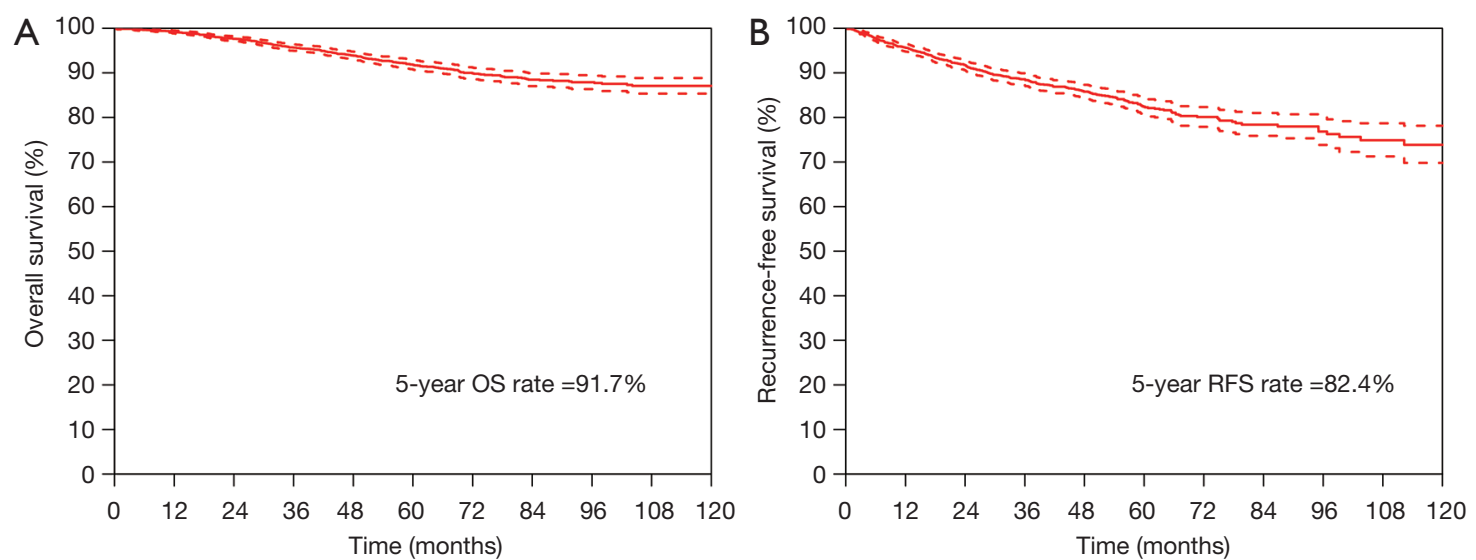

Figure 1 OS (left) and RFS (right) curves in resected stage I NSCLC. OS, overall survival; RFS, recurrence-free survival; NSCLC, nonsmall cell lung cancer.
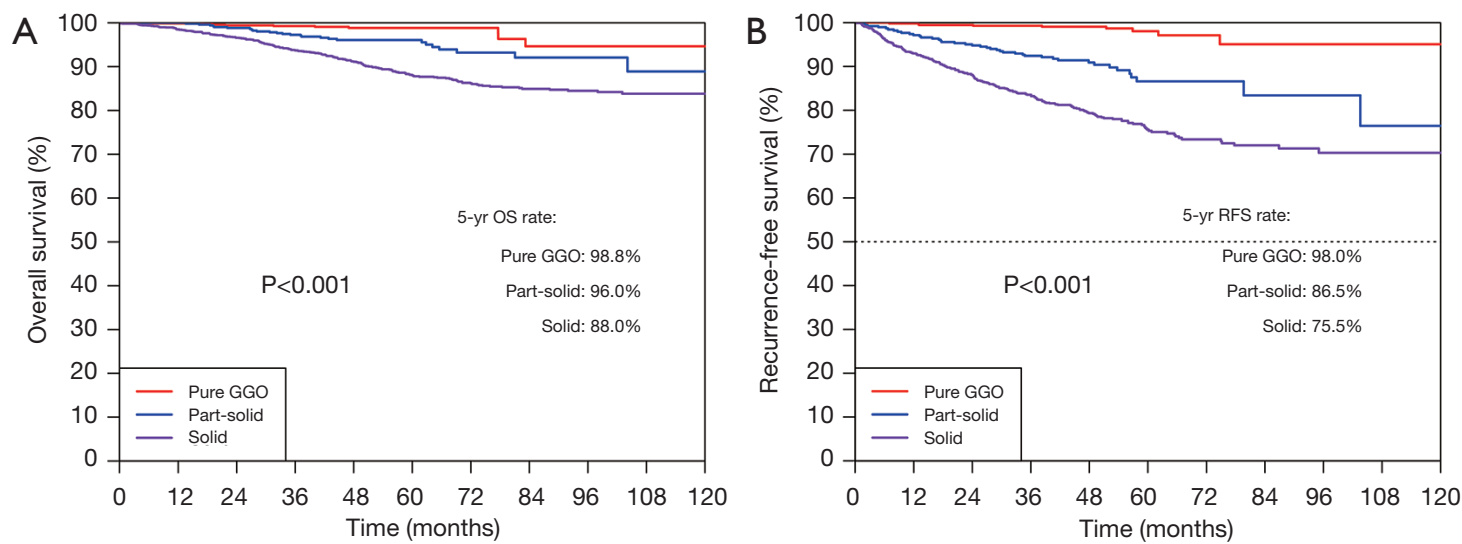

Figure 2 OS (left) and RFS (right) curves among the pure-GGO, part-solid, and solid groups in resected stage I NSCLC. OS, overall survival; RFS, recurrence-free survival; GGO, ground-glass opacity; NSCLC, non-small cell lung cancer.
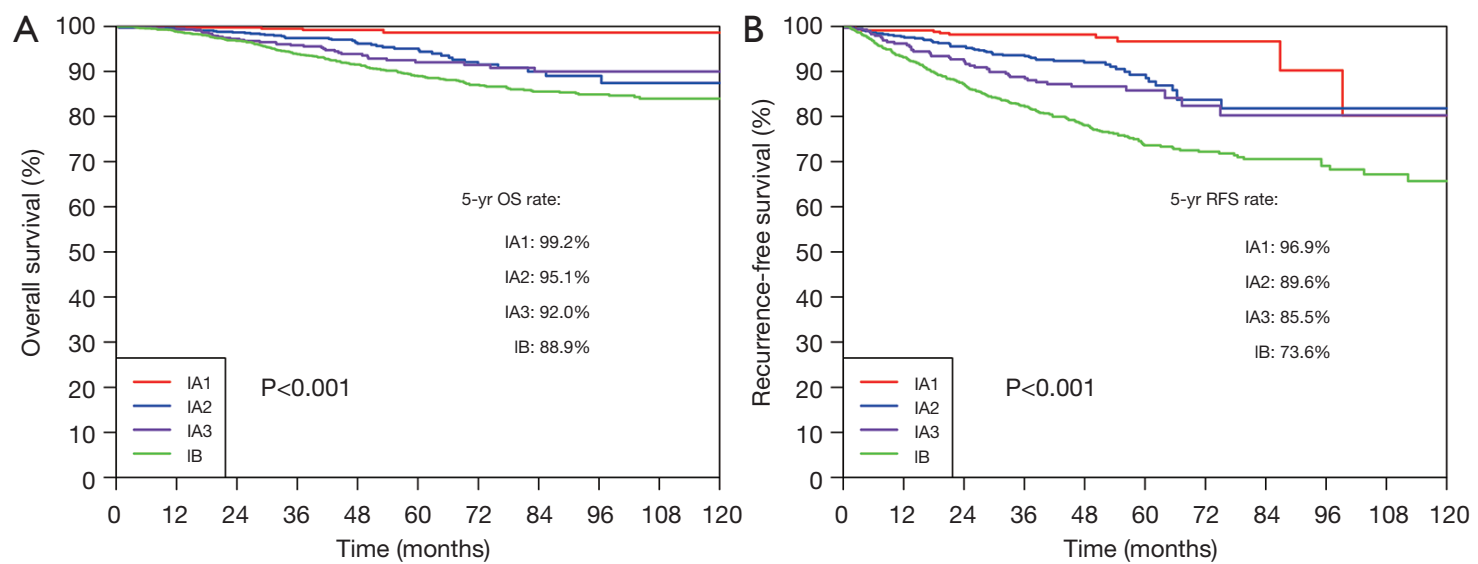

Figure 3 OS (left) and RFS (right) curves among the stage IA1/IA2/IA3/IB in resected NSCLC. OS, overall survival; RFS, recurrence-free survival; NSCLC, non-small cell lung cancer. 
Table 2 Univariate and multivariate Cox proportional hazards regression analysis of characteristics related with overall survival ( $\mathrm{n}=2,775$ )

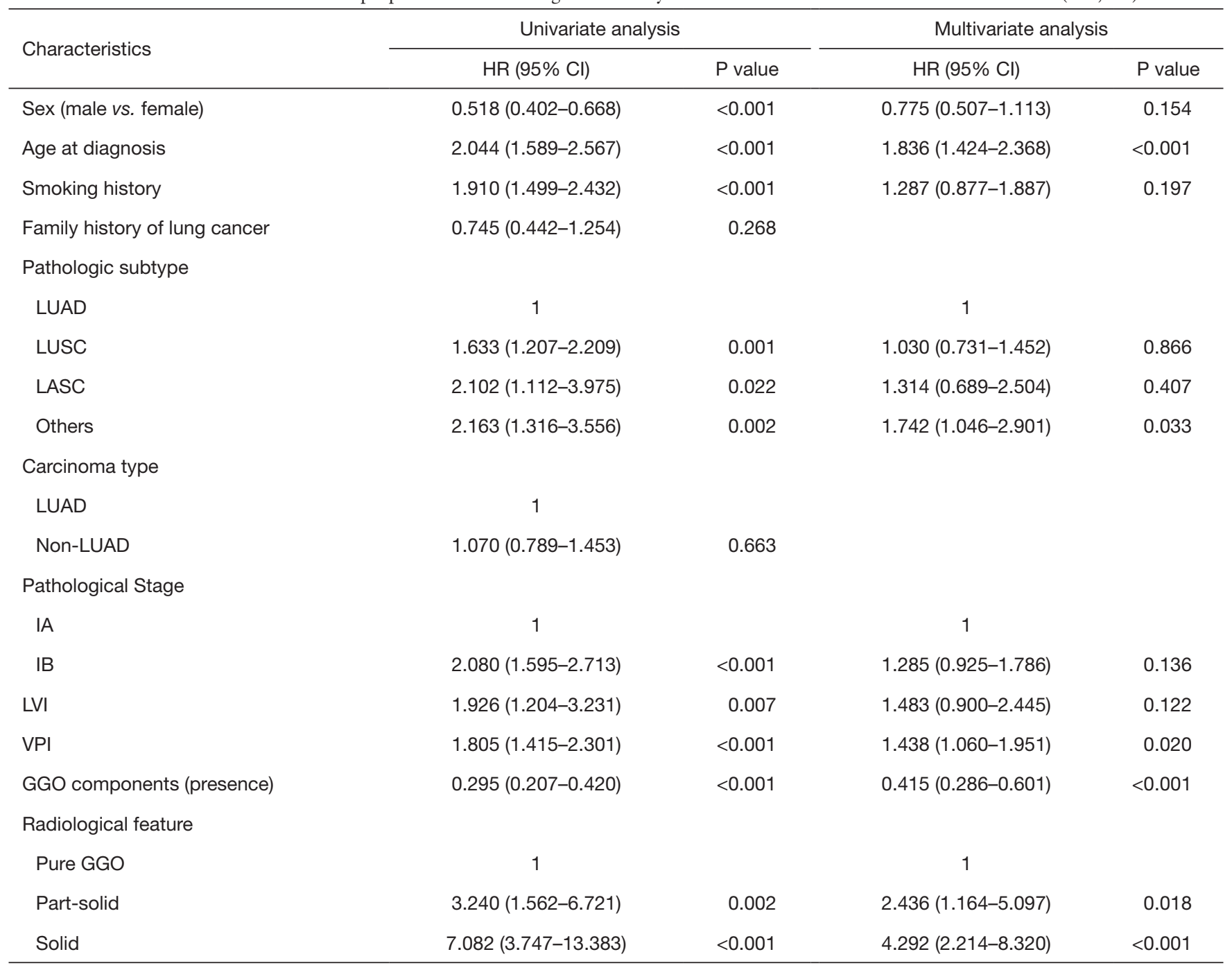

GGO, ground-glass opacity; LASC, lung adenosquamous carcinoma; LVI, lymphovascular invasion; LUAD, lung adenocarcinoma; LUSC, lung squamous carcinoma; VPI, visceral pleural invasion.

and solid group).

For patients with pure GGO nodules, stage IB (compared to IA) (HR $=9.070,95 \%$ CI: $1.560-52.749)$ and LVI (HR $=12.196$, 95\% CI: $1.333-111.570$ ) were independently associated with survival outcomes (Table 3). Although smoking history narrowly failed to achieve a statistical significance in the multivariate analysis, it was still suggested as a potential prognostic factor and worthy of further exploration (Table 3). For patients in the part-solid group, LVI (HR $=5.599,95 \%$ CI: 1.529-20.503) was independently associated with the survival (Table 4). Aside from LVI, sex (male vs. female, HR $=0.330,95 \%$ CI: 0.143-0.759,
$\mathrm{P}=0.009$ ), smoking history ( $\mathrm{HR}=3.825$, 95\% CI: $1.734-$ 8.438, $\mathrm{P}=0.001$ ) and non-LUAD subtypes (HR $=3.320,95 \%$ CI: $1.324-8.325, \mathrm{P}=0.010)$, LUSC in particular $(\mathrm{HR}=4.068$, 95\% CI: $1.523-10.865, \mathrm{P}=0.005)$ were found influential in the univariate analysis. But such differences were not achieved in the multivariate analysis (Table 4).

For patients with solid nodules, older age $(\mathrm{HR}=1.696$, 95\% CI: $1.280-2.248$ ), and VPI (HR $=1.467,95 \%$ CI: 1.041-2.066) were independent risk factors while female sex $(\mathrm{HR}=0.681,95 \%$ CI: 0.506-0.917) served as a predictor of better survival. The impact of clinical or pathological stage was shown to be significant on univariate analysis, but 
Table 3 Lung cancer survival by characteristics among patients with pure GGO nodules ( $\mathrm{n}=680$ )

\begin{tabular}{|c|c|c|c|c|}
\hline Characteristics & \multicolumn{2}{|c|}{ Univariate analysis } & \multicolumn{2}{|c|}{ Multivariate analysis } \\
\hline Sex (male vs. female) & $0.370(0.103-1.327)$ & 0.127 & & \\
\hline Age at diagnosis & $2.197(0.616-7.834)$ & 0.225 & & \\
\hline Smoking history & $4.151(1.198-14.381)$ & 0.025 & $3.526(0.981-12.681)$ & 0.054 \\
\hline \multicolumn{5}{|l|}{ Pathological stage } \\
\hline $\mathrm{IA}$ & 1 & & 1 & \\
\hline IB & $6.334(1.767-22.704)$ & 0.005 & $9.070(1.560-52.749)$ & 0.014 \\
\hline LVI & $9.511(1.199-75.460)$ & 0.033 & $12.196(1.333-111.570)$ & 0.027 \\
\hline
\end{tabular}

GGO, ground-glass opacity; LVI, lymphovascular invasion; VPI, visceral pleural invasion.

Table 4 Lung cancer survival by characteristics among patients with part-solid nodules $(\mathrm{n}=508)$

\begin{tabular}{|c|c|c|c|c|}
\hline Characteristics & \multicolumn{2}{|c|}{ Univariate analysis } & \multicolumn{2}{|c|}{ Multivariate analysis } \\
\hline Sex (male vs. female) & $0.330(0.143-0.759)$ & 0.009 & $0.857(0.205-3.580)$ & 0.833 \\
\hline Age at diagnosis & $1.187(0.865-4.046)$ & 0.112 & & \\
\hline Smoking history & 3.825 (1.734-8.438) & 0.001 & $2.806(0.705-11.173)$ & 0.143 \\
\hline \multicolumn{5}{|l|}{ Pathologic subtype } \\
\hline LUAD & 1 & & & \\
\hline LUSC & 4.068 (1.523-10.865) & 0.005 & & \\
\hline LASC & $2.904(0.382-22.058)$ & 0.303 & & \\
\hline Non-LUAD & $3.320(1.324-8.325)$ & 0.010 & $1.588(0.576-4.382)$ & 0.372 \\
\hline \multicolumn{5}{|l|}{ Pathological stage } \\
\hline IA & 1 & & & \\
\hline IB & $1.933(0.855-4.370)$ & 0.113 & & \\
\hline LVI & $8.857(2.586-30.335)$ & 0.001 & 5.599 (1.529-20.503) & 0.009 \\
\hline VPI & $1.296(0.587-2.858)$ & 0.521 & & \\
\hline
\end{tabular}

LASC, lung adenosquamous carcinoma; LVI, lymphovascular invasion; LUAD, lung adenocarcinoma; LUSC, lung squamous carcinoma; VPI, visceral pleural invasion. 
Table 5 Lung cancer survival by characteristics among patients with solid nodules $(\mathrm{n}=1,587)$

\begin{tabular}{|c|c|c|c|c|}
\hline Characteristics & \multicolumn{2}{|c|}{ Univariate analysis } & \multicolumn{2}{|c|}{ Multivariate analysis } \\
\hline Sex (male vs. female) & $0.699(0.521-0.937)$ & 0.017 & $0.681(0.506-0.917)$ & 0.011 \\
\hline Age at diagnosis & 1.765 (1.334-2.337) & $<0.001$ & $1.696(1.280-2.248)$ & $<0.001$ \\
\hline Smoking history & $1.270(0.959-1.681)$ & 0.095 & & \\
\hline \multicolumn{5}{|l|}{ Family history of cancer } \\
\hline Family history of lung cancer & $0.925(0.516-1.659)$ & 0.794 & & \\
\hline \multicolumn{5}{|l|}{ Pathologic subtype } \\
\hline LUAD & 1 & & & \\
\hline LUSC & $1.209(0.860-1.700)$ & 0.274 & & \\
\hline \multicolumn{5}{|l|}{ Carcinoma type } \\
\hline LUAD & 1 & & & \\
\hline Non-LUAD & $1.336(0.997-1.789)$ & 0.052 & & \\
\hline \multicolumn{5}{|l|}{ Clinical or pathological Stage } \\
\hline $\mathrm{IA}$ & 1 & & 1 & \\
\hline IB & $1.431(1.045-1.958)$ & 0.025 & $1.156(0.791-1.688)$ & 0.454 \\
\hline LVI & $1.155(0.642-2.076)$ & 0.631 & & \\
\hline VPI & $1.511(1.140-2.003)$ & 0.004 & $1.467(1.041-2.066)$ & 0.029 \\
\hline
\end{tabular}

LASC, lung adenosquamous carcinoma; LVI, lymphovascular invasion; LUAD, lung adenocarcinoma; LUSC, lung squamous carcinoma; VPI, visceral pleural invasion.

was reduced with the adjustment of confounding factors (Table 5).

Different groups had distinct prognostic factors. LVI was the shared risk factor for groups with presence of GGO components. Pathological stage (IA or IB) was influential exclusively for the pure-GGO group. In the solid group, females, younger patients, and patients without VPI had better survival. But such independent significance did not exist in the other two groups.

\section{Discussion}

The 8th edition of the TNM classification of NSCLC defines the $\mathrm{T}$ descriptors based on the radiological solid size or pathological invasive size and ignores the GGOfeatured component (17). Radiological solid size is consistent with the pathological invasive size in terms of patient survival (18). But GGO-featured components may not have such degree of malignancy comparable with that of solid components. The current study indicated that among patients with resected stage I lung cancer, as was consistent with known literature, GGO group had more favorable survival than both the part-solid group and the solid group either in the total cohort or in each $\mathrm{T}$ category (T1a, T1b, T1c, T2a). Prognostic factors were shown disparate between patients with GGO components and those without, which was also true between the part-solid and solid group $(15-16,19)$. Taking both the presence and the proportion of GGO components into account, there are 3 categories of patients: pure-GGO, part-solid and solid groups. Previous studies merely focused on two of the 3 groups or combined the pure-GGO group with part-solid group. As far as we know, the current study is the first to comprehensively investigate the distinct prognostic factors in all of the 3 groups parallelly in the resected stage I lung cancer. And a so-far largest cohort of patients $(n=2,775)$ were included. 
The proportions of various histological subtypes in the three groups may be different. In this study, LUAD (98.97\%) cases accounted for the largest proportion of the GGO group, and $91.48 \%$ of LUSC cases exhibited solid manifestation on CT scans. This supports the presumption that different pathological subtypes will have different survival outcomes depending on the presence of GGO, part-solid, or solid features. It was suggested that GGO components might serve as a confounding factor when researchers were looking into the impact of histological subtypes. Previous studies and this current study all confirmed the prognostic value of GGO components. Cho et al. revealed that the 5 -year OS rates for pure-GGO LUAD and mixed-GGO LUAD were $98.6 \%$ and $95.5 \%$ respectively (20), while Hattori et al. reported that the 5-year OS rate for the GGO-featured tumors seemed lower than that for solidfeatured lesions (95.3\% versus $96.8 \%$, respectively) (21). $\mathrm{Fu}$ et al. indicated that 5-year OS rates for pure-GGO, part-solid, and solid NSCLC were $98.5 \%, 94.9 \%$, and $83.4 \%$ respectively, and this difference was significant between the part-solid and solid groups but was negligible between the pure-GGO and part-solid groups (15). Our study was in line with these previous results. Therefore, we further investigated prognostic factors in the 3 groups separately.

Emerging evidences reveal that GGO-featured nodules have been demonstrated to influence the prognosis of resected patients in NSCLC $(13,19)$. In the current study, lung cancer with pure-GGO nodules had excellent OS and RFS. It was unlikely for patients in pure-GGO group to experience recurrence. In the total cohort, the 5-year RFS rate was $82.4 \%$ in this cohort, which was in line with our previous finding of the 5 -year overall recurrence rate for all stage I patients equal to $20.2 \%$ (22). But for pure-GGO patients, the 5-year RFS rate was $98.0 \%$. The necessity of lobectomy of pure-GGO-featured lung cancer is doubted and requires future investigation because of the excellent survival of these patients. Adjuvant chemotherapy might be a better choice.

Our results provide the evidence to the proposal that the next edition of TNM classification should focus on the importance of GGO components as a new $\mathrm{T}$ descriptor. However, there still remain several issues to be addressed. Pure-GGOs are relatively simple to assess, but no general consensus has not yet been reached concerning how to evaluate the extent of solid or GGO components in a part-solid nodule. Consolidation tumor ratio (CTR) was considered as the ratio of the maximum size of the solid component to the maximum tumor size on the CT scans, and CTRs for pure-GGO, part-solid, with solid nodules were $0,0-1$, and 1 respectively. This method may be too subjective to identify the specific solid diameter of complicated nodules with varying shapes. And how to associate and integrate the clinical stage and pathological stage is also not well defined. The pure-GGO, solid feature nodules accounts for the majority of lepidic and non-lepidic invasive LUAD cases respectively. It may be inappropriate to equate the diameter of solid nodules and ground-glass nodules in the evaluation of the $\mathrm{T}$ descriptor.

VPI was regarded as an important factor in TNM staging and was defined as a risk variable for prognosis $(23,24)$. Previous studies indicated that VPI was a significant independent factor in solid nodules but not in part-solid nodules $(15,25)$. Our study further demonstrated that VPI had no influence on either the part-solid group or pureGGO group but was a significant independent risk factor in the solid group $(\mathrm{P}=0.029)$. The solid group accounted for the majority of patients (1,587 in 2,775, 57.19\%), which was also consistent with previous studies. Due to the large population it might potentially impact, the role of VPI should be carefully considered in clinical practice.

LVI was defined as a tumor invasion which could affect prognosis. Previous studies reported that LVI was a significant risk factor in NSCLC patients with partsolid nodules $(\mathrm{P}=0.030)$ but not those with solid nodules ( $\mathrm{P}=0.054)$. Our study also found that LVI was strongly associated with prognosis in the part-solid group $(\mathrm{P}=0.009)$ and the pure-GGO group $(\mathrm{P}=0.027)$ but not in the solid group.

This study comprehensively examined the radiological and histologic characteristics in a relatively large cohort with a post-operative follow-up period of up to more than 80 months, and innovatively took both the presence and proportion of GGO components into consideration. However, it also had several limitations. Firstly, this study was retrospective in nature, and encountering study designrelated biases were inevitable. Secondly, this study was based on a single-institution and Chinese population with a high frequency of the never-smokers and LUAD patients. Thirdly, the sample size of the cohort may be limited, although the total of 2,775 patients was the largest cohort up to be enrolled for this type of study. Therefore, a larger population from multi-national centers in randomized controlled trials might be warranted to validate the prognostic value of the GGO components. 


\section{Conclusions}

GGO component was a prognosticator of favorable prognoses in stage I NSCLC patients. Prognostic factors and survival outcomes were disparate among the pureGGO, part-solid, and solid groups. Our results support the proposal that the next edition tumor-node-metastasis (TNM) classification should consider the importance of GGO components as a new T descriptor.

\section{Acknowledgments}

We would like to thank all the medical staff from West China Hospital of Sichuan University who have made contributions to the lung cancer database.

Funding: This project was supported by grants $(81871890$ and 91859203) from the National Natural Science Foundation of China, and a grant (2017-CY02-00030-GX) from the Science and Technology Project of Chengdu for Precision Medicine Research.

\section{Footnote}

Reporting Checklist: The authors have completed the STROBE reporting checklist. Available at http://dx.doi. org/10.21037/atm-20-4971

Data Sharing Statement: Available at http://dx.doi. org/10.21037/atm-20-4971

Conflicts of interest: All authors have completed the ICMJE uniform disclosure form (available at http://dx.doi. org/10.21037/atm-20-4971). The authors have no conflicts of interest to declare.

Ethical statement: The authors are accountable for all aspects of the work in ensuring that questions related to the accuracy or integrity of any part of the work are appropriately investigated and resolved. This study was approved by the Institutional Review Board of West China Hospital of Sichuan University (No. 2019.195). The study was conducted in accordance with the Declaration of Helsinki (as revised in 2013). Requirements of informed consent were waived due this study's retrospective nature.

Open Access Statement: This is an Open Access article distributed in accordance with the Creative Commons Attribution-NonCommercial-NoDerivs 4.0 International
License (CC BY-NC-ND 4.0), which permits the noncommercial replication and distribution of the article with the strict proviso that no changes or edits are made and the original work is properly cited (including links to both the formal publication through the relevant DOI and the license). See: https://creativecommons.org/licenses/by-nc-nd/4.0/.

\section{References}

1. Bray F, Ferlay J, Soerjomataram I, et al. Global cancer statistics 2018: GLOBOCAN estimates of incidence and mortality worldwide for 36 cancers in 185 countries. CA Cancer J Clin 2018;68:394-424.

2. Allemani C, Matsuda T, Di Carlo V, et al. Global surveillance of trends in cancer survival 2000-14 (CONCORD-3): analysis of individual records for 37513025 patients diagnosed with one of 18 cancers from 322 population-based registries in 71 countries. Lancet 2018;391:1023-75.

3. de Koning HJ, van der Aalst CM, de Jong PA, et al. Reduced lung-cancer mortality with volume CT screening in a randomized trial. N Engl J Med 2020;382:503-13.

4. National Lung Screening Trial Research Team, Aberle DR, Adams AM, et al. Reduced lung-cancer mortality with low-dose computed tomographic screening. $\mathrm{N}$ Engl J Med 2011;365:395-409.

5. De Koning, HJ, Van Der Aalst K, Ten Haaf M, et al. PL02.05 Effects of volume CT lung cancer screening: mortality results of the NELSON randomised-controlled population based trial. J Thorac Oncol 2018;13:S185.

6. Davies MPA, Cheng YI, Field JK, et al. Implementation planning for lung cancer screening in China. Precis Clin Med 2019;2:13-44.

7. Travis WD, Asamura H, Bankier AA, et al. The IASLC Lung Cancer Staging Project: proposals for coding T categories for subsolid nodules and assessment of tumor size in part-solid tumors in the forthcoming eighth edition of the TNM classification of lung cancer. J Thorac Oncol 2016;11:1204-23.

8. Brierly JD, Gospodarowicz MK, Wittekind Ch, editors. TNM Classification of Malignant Tumours. 8th ed. Hoboken, NJ: Wiley-Blackwell; 2017.

9. Aokage K, Miyoshi T, Ishii G et al. Influence of ground glass opacity and the corresponding pathological findings on survival in patients with clinical stage I non-small cell lung cancer. J Thorac Oncol 2018;13:533-42.

10. Walter JE, Heuvelmans MA, Bock GH, et al. Characteristics of new solid nodules detected in incidence 
screening rounds of low-dose CT lung cancer screening: the NELSON study. Thorax 2018;73:741-7.

11. Horeweg N, van Rosmalen J, Heuvelmans MA, et al. Lung cancer probability in patients with CT-detected pulmonary nodules: a prespecified analysis of data from the 1. NELSON trial of low-dose CT screening. Lancet Oncol 2014;15:1332-41.

12. McWilliams A, Tammemagi MC, Mayo JR, et al. Probability of cancer in pulmonary nodules detected on first screening CT. N Engl J Med 2013;369:910-9.

13. Hattori A, Matsunaga T, Takamochi K, et al. Prognostic impact of a ground glass opacity component in the clinical T classification of non-small cell lung cancer. J Thorac Cardiovasc Surg 2017;154:2102-10.e1.

14. Hattori A, Matsunaga T, Takamochi K, et al. Importance of ground glass opacity component in clinical stage IA radiologic invasive lung cancer. Ann Thorac Surg 2017;104:313-20.

15. Fu F, Zhang $\mathrm{Y}$, Wen $Z$ et al. Distinct prognostic factors in patients with stage I non-small cell lung cancer with radiologic part-solid or solid lesions. J Thorac Oncol 2019;14:2133-42.

16. Ye T, Deng L, Wang S, et al. Lung adenocarcinomas manifesting as radiological part-solid nodules define a special clinical subtype. J Thorac Oncol 2019;14:617-27.

17. Rami-Porta R, Bolejack V, Crowley J, et al. The IASLC lung cancer staging project: proposals for the revisions of the $\mathrm{T}$ descriptors in the forthcoming eighth edition of the TNM classification for lung cancer. J Thorac Oncol 2015;10:990-1003.

18. Aokage K, Miyoshi T, Ishii G, et al. Clinical and pathological staging validation in the eighth edition of the TNM classification for lung cancer: correlation between solid size on thin-Section computed tomography

Cite this article as: Wang $\mathrm{C}, \mathrm{Wu} \mathrm{Y}, \mathrm{Li}$ J, Ren P, Gou Y, Shao J, Zhou Y, Xiao X, Tuersun P, Liu D, Zhang L, Li W. Distinct clinicopathologic factors and prognosis based on the presence of ground glass opacity components in patients with resected stage I non-small cell lung cancer. Ann Transl Med 2020;8(18):1133. doi: 10.21037/atm-20-4971 and invasive size in pathological findings in the new $\mathrm{T}$ classification. J Thorac Oncol 2017;12:1403-12.

19. Hattori A, Hirayama S, Matsunaga T, et al. Distinct clinicopathologic characteristics and prognosis based on the presence of ground glass opacity component in clinical stage IA lung adenocarcinoma. J Thorac Oncol 2019;14:265-75.

20. Cho JH, Choi YS, Kim J, et al. Long-term outcomes of wedge resection for pulmonary ground-glass opacity nodules. Ann Thorac Surg 2015;99:218-22.

21. Hattori A, Matsunaga T, Takamochi K, et al. Importance of ground glass opacity component in clinical stage IA radiologic invasive lung cancer. Ann Thorac Surg 2017;104:313-20.

22. Wang C, Wu Y, Shao J, et al. Clinicopathological variables influencing overall survival, recurrence and postrecurrence survival in resected stage I non-small-cell lung cancer. BMC Cancer 2020;20:150.

23. Yoshida J, Nagai K, Asamura H, et al. Visceral pleura invasion impact on non-small cell lung cancer patient survival: its implications for the forthcoming TNM staging based on a large-scale nation-wide database. J Thorac Oncol 2009;4:959-63.

24. Butnor KJ, Travis WD. Recent advances in our understanding of lung cancer visceral pleural invasion and other forms of minimal invasion: implications for the next TNM classification. Eur J Cardiothorac Surg 2013;43:309-11.

25. Hattori A, Suzuki K, Matsunaga T, et al. Visceral pleural invasion is not a significant prognostic factor in patients with a part-solid lung cancer. Ann Thorac Surg 2014;98:433-8.

(English Language Editor: J. Gray) 